\title{
Kwok-ying Lau, Phenomenology and Intercultural Understanding: Toward a New Cultural Flesh
}

Séries «Contributions to Phenomenology », $n^{\circ} 87$, Dordrecht, Springer, 2016, xii +256 pages

\section{Jean-Claude Gens}

\section{(2) OpenEdition}

\section{Journals}

Édition électronique

URL : http://journals.openedition.org/alter/704

DOI : $10.4000 /$ alter.704

ISSN : 2558-7927

Éditeur :

Association ALTER, Archives Husserl (CNRS-UMR 8547)

\section{Édition imprimée}

Date de publication : 31 décembre 2018

Pagination : 261-266

ISBN : 978-2-9550449-4-0

ISSN : 1249-8947

\section{Référence électronique}

Jean-Claude Gens, « Kwok-ying Lau, Phenomenology and Intercultural Understanding : Toward a New Cultural Flesh », Alter [En ligne], 26 | 2018, mis en ligne le 22 janvier 2019, consulté le 23 septembre 2020. URL : http://journals.openedition.org/alter/704 ; DOI : https://doi.org/10.4000/alter.704 


\section{KWOK-YING LAU, PHENOMENOLOGY AND INTERCULTURAL UNDERSTANDING : TOWARD A NEW CULTURAL FLESH, SÉRIES «CONTRIBUTIONS TO PHENOMENOLOGY », $\mathrm{N}^{\circ} 87$, DORDRECHT, SPRINGER, 2016, XII + 256 PAGES}

Jean-Claude Gens

Initialement écrits à l'occasion de conférences données entre 1996 et 2016 en Asie, aux Amériques et en Europe, les onze chapitres de ce volume interrogent la possibilité, en partant de la phénoménologie, d'engager un dialogue interculturel entre l'Europe et l'Orient, et, en l'occurrence, la Chine. Comme l'indique déjà le sous-titre, l'auteur invite en fin de compte, pour entrer en un tel dialogue, à une nouvelle appropriation du concept merleau-pontien de chair, ce qui le conduit à forger l'expression originale et, au premier abord, étonnante de « chair culturelle » à laquelle sont en particulier consacrés les deux derniers chapitres.

Plutôt introductifs, les deux premiers chapitres questionnent l'idée même de compréhension interculturelle en pointant les limites de l'idée de la philosophie telle que la conçoit la Krisis ; à la conception d'une identité simple et non problématique de l'Europe, l'auteur oppose à la fois le constat de Lévi-Strauss selon lequel toute culture, y compris celle de l'Europe, est le fruit d'hybridations - mais ce constat relatif à la réalité empirique des cultures se situe-t-il sur le même plan que l'Idée de l'Europe ? - d'une part, et la suggestion merleau-pontienne selon laquelle la découverte du propre de notre culture suppose l'expérience d'un décentrement, d'une aliénation, ou encore d'un « se laisser enseigner par une autre culture », pour reprendre l'expression de Signes $^{1} \mathrm{~d}^{\prime}$ autre part ; et cela vaut aussi évidemment de la philo-

${ }^{1}$ M. Merleau-Ponty, Signes, Paris, Gallimard, 1960, p. 151. 
sophie, sauf à vouloir s'arcbouter sur son essence "grecque », ce qui implique à la fois l'oubli que «la » Grèce est elle-même le fruit d'un certain nombre de rencontres, et la réduction de la philosophie à une spécialité culturelle comme le camembert est une spécialité de Normandie, ou la soupe Pho une spécialité vietnamienne.

La suite de l'ouvrage a le mérite de ne pas en rester à des réflexions générales puisque, dans une perspective toujours critique à l'égard de l'européocentrisme, elle commence par inviter à une lecture phénoménologique du Daodejing. La difficulté de ce genre d'entreprise, c'est d'éviter la juxtaposition abstraite de notions dont le rapprochement est d'autant plus facile qu'on les a par avance coupées du réseau des autres notions à l'intérieur desquelles, seulement, elles prennent leur véritablement sens. Le plus fécond et le plus concret, à mon sens, c'est comme y invite par exemple Heidegger - de reconduire ces notions à des expériences primitives qu'elles ont, à un moment donné, cherché à dire ; c'est manifestement ce dont part aussi Jean-François Billeter en faisant l'hypothèse que le Zhuangzi renvoie à des expériences, au fond, universelles.

$C^{\prime}$ est dans ce cadre qu'il conviendrait de comprendre une des suggestions de ce chapitre trois relatif à la possibilité de lire phénoménologiquement le Daodejing : ne serait-il pas possible d'éclairer l'une par l'autre les notions de Dao et de nature primordiale à laquelle MerleauPonty invite à rétrocéder et plus clairement encore à ce qu'il appelle l'être sauvage - une nature antérieure à la distinction sujet-objet (p. 45) et dont l'être sauvage est à l'antipode de la nature que nous avons réduit à l'état de ressource (p. 51 sq.).

Le caractère plus exploratoire que systématique de l'ouvrage s'atteste au regard du fait que si le chapitre 4 s'arrête au bouddhisme, dont on sait qu'il constitue avec le confucianisme l'une des trois grandes écoles en Chine, $c^{\prime}$ est seulement à propos de la compréhension que Husserl a pu en avoir et dont témoigne en particulier sa recension de 1925 «Über die Reden Gotamo Buddhos ». À cet égard, il est néanmoins remarquable que loin d'en rester au constat du caractère en fin de compte européocentrique et donc limité de la pensée husserlienne qui contraste avec l'ouverture manifeste de Heidegger à l'égard de la culture extrême-orientale, l'auteur lit, comme MerleauPonty, Husserl de manière généreuse. Si Merleau-Ponty salue en Husserl celui qui, «bon gré mal gré, contre ses plans et selon son audace essentielle, [...] réveille un monde sauvage et un esprit sauvage $»^{2}$, Lau pointe aussi - malgré ce qui les sépare - la similarité des

${ }^{2}$ M. Merleau-Ponty, Signes, op. cit., p. 228. 
Kwok-ying Lau, «Phenomenology and Intercultural Understanding »

gestes husserlien et taoïste invitant à revenir à l'origine pour permettre un renouveau (p. 52).

Les chapitres 5 et 6 élargissent la perspective en montrant dans quelle mesure la phénoménologie de Patočka est, comparée aux textes de la Krisis, non européocentrique et en amorçant une discussion possible entre ce philosophe pragois et Mencius à propos de la question du soin de l'âme. À l'issue de ces rencontres avec chacune des trois grandes écoles - le taoïsme, le bouddhisme et le confucianisme -, les chapitres suivants interrogent de manière plus globale mais toujours très précisément les limites de l'européocentrisme, la question centrale du souci de soi et les conditions effectives d'un dialogue interculturel.

Le chapitre 7 oppose à la naïveté de l'européocentrisme - mais cela vaut aussi , comme il le précise, du sinocentrisme - la thèse de LéviStrauss mettant l'accent sur le caractère hybride des cultures, et Lau met en ce sens en évidence deux facteurs qui ont contribué à l'avènement du désenchantement du monde en Occident: la controverse relative à la chronologie chinoise, qui laisse entendre que les commencements de la culture chinoise seraient antérieurs à l'origine de l'histoire telle qu'elle était calculée à partir du récit de la Genèse, et la controverse relative à la signification religieuse ou purement sociale des rites aux XVII et XVIII's siècles; autrement dit, ce seraient deux facteurs de la constitution de l'identité de la culture occidentale moderne.

Le chapitre 8 revient de manière plus approfondie sur la question du soin de l'âme en considérant aussi bien les leçons de Foucault relatives aux techniques de soi que la pensée d'un philosophe chinois contemporain moins connu en Occident : Lao Sze-Kwang (1927-2012). Auteur d'une histoire de la philosophie chinoise en trois volumes, Lao défend, contre la dimension essentiellement cognitive ou théorique de la philosophie occidentale, une philosophie qu'il qualifie $\mathrm{d}^{\prime}$ " orientative ». Celle-ci ne rejette pas cette dimension théorique et argumentative, mais la considère comme devant servir une visée pratique de transformation de soi et du monde ; dit en des termes grecs classiques, la connaissance de soi, à laquelle invitait déjà Socrate, n'a de sens que par rapport au souci de soi.

La référence de Lao aussi bien à Zhuangzi qu'à Mencius et au néoconfucéen Wang Yangming montre ce que, par-delà tout ce qui les sépare, ils ont en commun: le souci de penser et de réaliser une transformation pratique de soi. Si, comme le dit Lau, ce qui intéresse Lao, c'est « où devrions-nous aller ? » plutôt que " qu' est-ce que $c^{\prime}$ est ? » (p. 135), on comprend d'autant mieux la facilité de la réception du pragmatisme américain en Chine. Certes, les conférences de Dewey 
durant son séjour de deux années en Chine (1919-1920) ont contribué à cette réception, et Lao est aussi tout à fait susceptible de l'avoir lu ; autrement dit, la conception de la philosophie chinoise selon Lao consonne avec l'invitation de James à considérer la question de savoir ce qu'il est possible d'espérer d'une chose, d'un être ou d'un moment donné, ce qu'elle ou il promet, plutôt que de se soucier de savoir question platonicienne - quelle en serait la «nature » ou l'essence. Comme le tournant éthique auquel invitent Foucault dans ses derniers cours ou Richard Rorty, l'idée d'une philosophie orientative est par ailleurs évidemment critique à l'égard de l'idée selon laquelle il faudrait distinguer la philosophie en tant que pure theoria d'une part, de la sagesse d'autre part, à laquelle les pensées chinoise ou indienne sont reconduites par Hegel, Husserl et, dans leur foulée, par François Jullien (p. 213 note).

Lau corrèle au chapitre 9 cette conception de la philosophie comme orientative aux cours de Foucault relatifs aux pratiques de soi. On pourrait néanmoins se demander d'une part si ce n'est pas du côté de la conception que Pierre Hadot se fait de ce souci de soi que la résonance avec une dimension ou un courant majeur de la pensée chinoise serait plus patente encore, et d'autre part si cette dimension orientative de la philosophie n'est pas aussi bien mise en avant par un autre philosophe chinois contemporain qui ne fait pas pour autant usage du qualificatif d'orientatif : Mou Zongsan. Sans faire usage du qualificatif d'orientatif, ses conférences traduites en français sous le titre de Spécificités de la philosophie chinoise (2003) pointent la proximité de la philosophie chinoise par exemple avec les philosophies de l'existence et de la vie.

Ce qui est en réalité en jeu, c'est la possibilité de parler au singulier de la philosophie chinoise dans la mesure où, nourris tous deux de la pensée kantienne dont ils proposent une lecture différente, Mou et Lao s'inscrivent dans des traditions de pensée divergentes, le premier accordant, à la différence du second, une importance majeure à la dimension cosmique de l'existence, et donc aussi au courant cosmologique de la pensée chinoise traditionnelle. Cette question n'est pas sans rapport avec la première : Lau cite en passant un court passage de Hadot selon lequel la sagesse telle que l'entendait la philosophie antique devait permettre $d^{\prime}$ atteindre non seulement l'ataraxie, l'autarcie, mais encore une « conscience cosmique » (p. 146). Ce que pointe en effet Hadot, tout en renvoyant à une expérience personnelle effective, c'est un soi qui demande à être conçu et vécu dans un rapport essentiel à la nature - une dimension pointée aussi dans la pensée confucéenne, par exemple dans l'idée selon laquelle l'homme doit 
Kwok-ying Lau, «Phenomenology and Intercultural Understanding »

s'efforcer de constituer une trinité avec le Ciel et la terre, et, plus récemment, par Arne Naess dans La réalisation de soi, mais absente chez Foucault. La corrélation très pertinente entre Lao et Foucault pourrait en ce sens trouver son contrepoint dans une corrélation entre Pierre Hadot et Mou Zongsan.

Le chapitre 10 intitulé «La chair : de son usage ontologique à un usage interculturel » entre plus décisivement dans une interrogation de cette notion de chair. Rappelant que le concept de chair s'esquisse déjà dans La structure du comportement lorsque Merleau-Ponty pointe la plus grande originarité de la nature primordiale en tant que champ sensible pré-objectif, par rapport à la «nature » des sciences de la nature ${ }^{3}$, Lau défend la thèse selon laquelle le caractère ambigu de la notion merleau-pontienne de chair n'est pas une objection contre sa pertinence comme on l'a parfois pensé, mais est au contraire ce qui en fait toute la consistance (p. 178). Quel est l'intérêt d'une telle notion au regard de la question initiale relative à la compréhension interculturelle?

S'il nous faut d'autant plus promouvoir une telle compréhension après un événement comme celui du 11 septembre, avance Kwok-ying Lau, celle-ci suppose de pouvoir entrer dans la Stimmung ou la tonalité culturelle de la culture qu'il s'agit de comprendre. Or, pour y parvenir, il est nécessaire de "s'autogreffer » une nouvelle chair culturelle (p. 190), c'est-à-dire de « transplanter » (p. 191) pour ainsi dire en nous la sensibilité culturelle de l'autre, de se laisser habiter par sa sensibilité culturelle. La thèse est relativement proche de celle que défend Rorty lorsqu'il déclare que l'éducation morale susceptible d'empêcher effectivement l'avènement de phénomènes comme le nazisme ou la guerre de Yougoslavie est nécessairement une éducation de la sensibilité. À ses yeux, c'est la littérature et le cinéma, y compris le documentaire, qui ont le pouvoir de nous faire percevoir comme un semblable celui qui diffère néanmoins radicalement de nous par sa façon de parler, de se nourrir, de se vêtir...

L'originalité à cet égard de la thèse de Lau, c'est non seulement de s'appuyer sur une ontologie merleau-pontienne qui met l'accent sur la dimension intersubjective, c'est-à-dire intercorporelle du monde, la dimension intermondaine $\mathrm{du}$ monde, mais d'aller au bout de cette logique en suggérant qu'entrer dans la Stimmung de l'autre, c'est apprécier ses œuvres d'art, sa musique, son théâtre..., se nourrir comme il le fait, savourer ses plats et ses vins, et même s'habiller comme lui. Il ne s'agit évidemment pas de penser que la différence se

${ }^{3}$ M. Merleau-Ponty, La structure du comportement, Paris, PUF, 1972, p. 187, 180, note. 
verrait ainsi abolie ; mais c'est là un moyen de se laisser toucher par un autre mode d'être, c'est-à-dire de penser et de sentir. C'est en ce sens que doit s'entendre l'idée, immédiatement obscure, d'une culture nécessaire de "l'esprit culturel sauvage » (cultural savage mind) - une expression vouée à rester obscure tant que l'on en reste au dualisme scolaire entre la nature et la culture, sans percevoir que la nature «transparait » toujours dans la culture, comme dit la Phénoménologie de la perception ${ }^{4}$. Autrement dit, il s'agit de développer en soi la sensibilité aux autres «par l'enroulement de leur sensibilité culturelle sur la nôtre » (p. 190).

Le chapitre 11 poursuit l'interrogation des conditions de possibilité d'un dialogue interculturel en mettant en évidence la façon dont les travaux de Lévi-Strauss ont nourri la pensée de Merleau-Ponty en particulier en ce qui concerne sa notion d'une universalité latérale et plus largement ses réflexions relatives à ce qu'il appelait la « littérature pensante » de l'Orient. Le dernier chapitre répond enfin à la question de savoir dans quelle mesure la notion merleau-pontienne de chair et plus largement la conceptualité dont elle est indissociable (les notions d'intercorporéité, d'intermonde, de réversibilité...) est somme toute la plus à même de permettre de penser et de mettre en œuvre un dialogue interculturel comparée aux conceptualités propres à d'autres figures de la phénoménologie, celles de Levinas, de Derrida ou de Henry, ou à celle de Deleuze ; la perspective serait probablement différente en ce qui concerne un autre phénoménologue, lui, plus proche de MerleauPonty : Henri Maldiney.

Partant le plus souvent de la phénoménologie pour chercher à discuter avec la pensée chinoise, sauf lorsqu'il part de Lao, ce livre donne donc d'autant plus à penser qu'il invite en fin de compte à considérer les différentes voies en lesquelles s'est engagée la phénoménologie du point de vue du défi que représente aujourd'hui la nécessité d'entrer dans un dialogue interculturel. Mais, en forgeant la notion de chair culturelle, Kwok-ying Lau entreprend à la fois une relecture appropriante de Merleau-Ponty, qui met en évidence le caractère incontournable de sa pensée tout en la prolongeant, et une interrogation relative à la possibilité de surmonter effectivement la naïveté de l'ethnocentrisme de la pensée occidentale, et plus largement de la pensée tout court.

${ }^{4}$ M. Merleau-Ponty, Phénoménologie de la perception, Paris, PUF, 1945, p. 339. 